\title{
Rapid and Simple Method for the Observation with Scanning Electron Microscopy of Blumenbachia Insignis Schrad.
}

Oppedisano, M.A. ${ }^{*}$, Cardinali, F.J. ${ }^{2-3}$, Thevenon, M.A ${ }^{2}$, Burgos Herrera, ${ }^{2}$

${ }^{1}$ Laboratorio de Microscopia Electrónica, Universidad Nacional de Mar del Plata. Argentina

${ }^{2}$ Laboratorio de Botánica, Facultad de Ciencias Exactas y Naturales, Universidad Nacional de Mar del Plata. Argentina.

${ }^{3}$ Laboratorio de, Fisiología VegetalFacultad de Ciencias Agrarias, Universidad Nacional de Mar del Plata. Argentina.

* Corresponding author: microsc@ mdp.edu.ar

Blumenbachia insignis Schrad. (Ortiga macho) is a species native to Argentina, used in folk medicine as anti-rheumatic by way of infusions of its aerial organs. A scarce morpho-anatomic information on its seed and fatty acid content is available.

The plant tissues involve a careful processing to be observed with SEM, spending a considerable time for fixing, dehydration and drying, as well as the use of specific and expensive reagents.

The objective of this work is to report a simple methodology for the observation with SEM of samples maintained in FAA, in order to obtain morpho-anatomic information of the seed, and to know the content of fatty bodies.

Dry whole seeds of Blumenbachia insignis Schrad. were preserved in FAA, and processed using the rapid method of inclusion in paraffin. Cuts were made in a Minot microtome, and mounted on slides to be de-waxed. The slides were glued on aluminium supports for metallization, and observed with SEM. The applied protocol allowed to obtain excellent images of seed, embryonic and reserve tissues, and a clear observation of cell walls and lipid bodies of different sizes.

The speed and economy of the procedure facilitated the morpho-anatomic characterization of this species. 


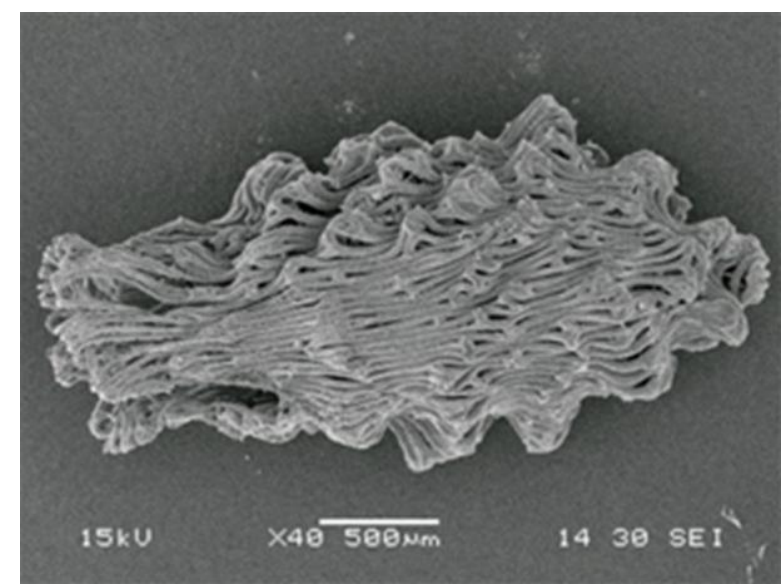

Fig. 1. Whole seed view.

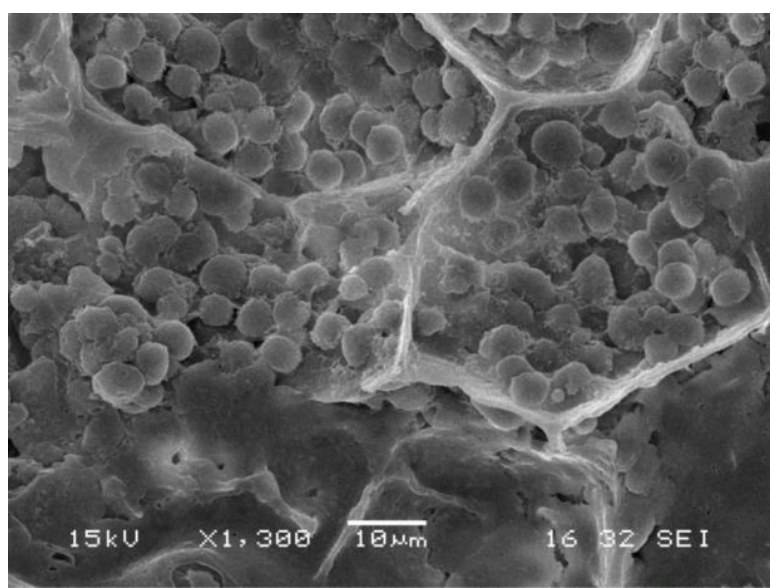

Fig. 2. Endosperm parenchymal cells with abundant lipid bodies and conspicuous cell walls 